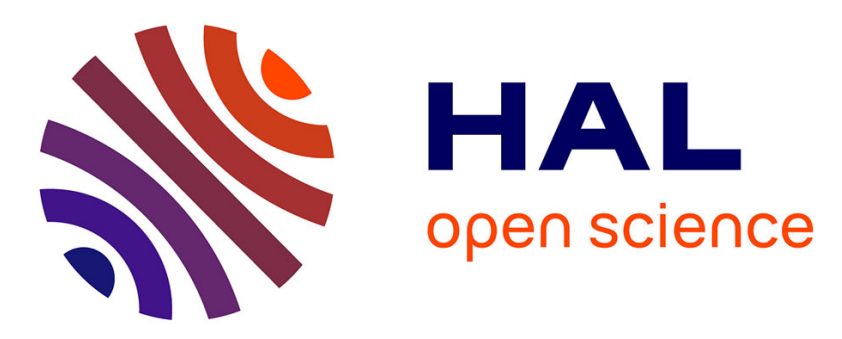

\title{
A robust stability framework for LTI systems with time-varying sampling
}

Christophe Fiter, Laurentiu Hetel, Wilfrid Perruquetti, Jean-Pierre Richard

\section{To cite this version:}

Christophe Fiter, Laurentiu Hetel, Wilfrid Perruquetti, Jean-Pierre Richard. A robust stability framework for LTI systems with time-varying sampling. Automatica, 2015, 54, pp.56-64. 10.1016/j.automatica.2015.01.035 . hal-01194561

\section{HAL Id: hal-01194561 \\ https://inria.hal.science/hal-01194561}

Submitted on 7 Sep 2015

HAL is a multi-disciplinary open access archive for the deposit and dissemination of scientific research documents, whether they are published or not. The documents may come from teaching and research institutions in France or abroad, or from public or private research centers.
L'archive ouverte pluridisciplinaire HAL, est destinée au dépôt et à la diffusion de documents scientifiques de niveau recherche, publiés ou non, émanant des établissements d'enseignement et de recherche français ou étrangers, des laboratoires publics ou privés. 


\title{
A Robust Stability Framework for LTI Systems with Time-Varying Sampling *
}

\author{
Christophe Fiter ${ }^{a, *}$ Laurentiu Hetel ${ }^{\text {a }}$ Wilfrid Perruquetti ${ }^{\mathrm{a}, \mathrm{b}}$ Jean-Pierre Richard ${ }^{\mathrm{a}, \mathrm{b}}$ \\ ${ }^{a}$ Centre de Recherche en Informatique, Signal et Automatique de Lille (CRIStAL, CNRS UMR 9189), \\ Université Lille 1 / École Centrale de Lille, 59650 Villeneuve d'Ascq, France \\ ${ }^{\mathrm{b}}$ Non-A, INRIA Lille-Nord Europe, France
}

\begin{abstract}
This work aims at enlarging the sampling intervals in several state feedback control situations by designing a sampling map in the state space. We consider the case of linear time invariant (LTI) systems with state-bounded perturbations, and guarantee their exponential stability for a chosen decay-rate. The approach is based on linear matrix inequalities (LMIs) obtained thanks to Lyapunov-Razumikhin stability conditions and convexification arguments. First, it enables to optimize the lower-bound of the sampling maps by computing the adequate Lyapunov-Razumikhin function. This result can be interpreted as a robust stability analysis with respect to arbitrary time-varying sampling intervals, which may be useful in the case of uncontrolled sampling, or in the presence of phenomenon such as sampling jitter. Then, the obtained results are extended to design the sampling map in three dynamic sampling control situations: event-triggered control, self-triggered control, and state-dependent sampling. The results are illustrated with a numerical example from the literature.
\end{abstract}

Key words: state-dependent sampling, self-triggered control, event-triggered control, time-varying sampling, linear matrix inequality, Lyapunov-Razumikhin, convex embedding, sampled-data control, sampling jitter

\section{Introduction}

Networked Control Systems are often required to share limited communication and computation resources, which leads to fluctuations of the sampling interval. From the control theory point of view, these variations in the sampling bring up new challenges. In the literature, two main research lines consider this aspect.

Stability analysis under arbitrary time-varying sampling: In the past decade, several works concerning the robust stability analysis of sampled-data systems with arbitrary time-varying sampling have been proposed (see [10] for a short survey), based on Impulsive delay differential Equations [34,12,11,20,32], on Input-Output approaches $[26,13,28]$, on Hybrid approaches [27,18], and on Discrete-time / Convex embeddings [16,5,17].

\footnotetext{
* The research leading to these results has received funding from the ANR project ROCC-SYS (ANR-14-CE27-0008), the Région Nord - Pas de Calais project ARCIR ESTIREZ, and the European Community's Seventh Framework Programme (FP7) HYCON2 (grant agreement $\mathrm{n}^{\circ} 257462$ ).

* Corresponding author.

Email address: christophe.fiter@univ-lille1.fr (Christophe Fiter).
}

Various numerical benchmarks [8] show that convex embeddings often lead to less conservative results than most approaches in the case of linear systems. This is due to the fact that its conservatism can be traded in exchange for computational complexity. However, considering unknown exogenous disturbances using that approach is not an easy task, and to our knowledge, no such study has yet been performed.

Dynamic sampling control: Recently, intensive research has also been conducted to control dynamically the sampling in order to reduce the processor and/or network loads while ensuring the desired control performances. There are several approaches in the literature:

- Event-triggered control [33,23,31,6]: intelligent sensors are used to monitor the state continuously and send information to the controller when special events occur. This requires a dedicated hardware.

- Self-triggered control [35,36,25,1,2]: it does as eventtriggered control without dedicated hardware, by estimating (online) the next admissible sampling interval. - Periodic event-triggered control [3,15,30,37,29]: it is an event-triggered control scheme in which the system's state is monitored periodically instead of continuously, which also allows for avoiding dedicated hardware. 
- State-dependent sampling [8]: the approach is a combination between robust stability analysis with respect to arbitrary time-varying sampling, and self-triggered control. The idea is to consider a partition of the state space into conic regions and to compute on each of them, offline, the maximum allowable sampling interval so as to design a sampling map. An optimisation of the lower-bound of the sampling map is possible. Up to now, only ideal LTI systems were considered.

In the present work, we consider the case of perturbed LTI systems, with an unknown exogenous disturbance supposed to be state-bounded, and we provide tools to perform robust stability analysis regarding time-varying sampling, event-triggered control, self-triggered control, and state-dependent sampling. For each of these applications, we ensure the system's exponential stability for a given decay-rate $\beta$, thanks to Lyapunov-Razumikhin stability conditions and convexification arguments.

The main contributions w.r.t. the literature are:

- The consideration of an unknown exogenous disturbance with convex embedding techniques in a robust stability analysis regarding time-varying sampling.

- The optimization of the lower-bound of the sampling map obtained with event-triggered and self-triggered control schemes. In particular, we use a robust optimization based on Lyapunov-Razumikhin Function (LRF) which takes into account both the effects of the sampling and the perturbation when designing the sampling map. - The design of a state-dependent sampling scheme for the case of perturbed LTI systems.

- The consideration of potential uncertainties or sampling jitter for dynamic sampling schemes.

- A new event-triggered control scheme in which continuous monitoring of the system's state is necessary only after a certain lapse of time.

The paper is organized as follows. First, we state the problem in Section 2 and propose a grounding stability result in Section 3. Then, Sections 4 to 7 present the robust stability analysis regarding time-varying sampling and the design of the different dynamic sampling control schemes. Finally, some simulation results are shown in Section 8 before concluding in Section 9. All the proofs and a technical lemma are given in Appendix 10.

Notations: $\mathbb{R}_{+}=\{\lambda \in \mathbb{R}, \lambda \geq 0\}, \mathbb{R}^{*}=\mathbb{R} \backslash\{0\}$, and $\mathcal{M}_{n}(\mathbb{R})=\mathbb{R}^{n \times n} . \mathbb{S}_{n}^{+}$(resp. $\mathbb{S}_{n}^{+*}$ ) is the set of positive (resp. positive definite) symmetric matrices $P \succeq 0$ (resp. $P \succ 0)$ in $\mathcal{M}_{n}(\mathbb{R})$. The largest (resp. lowest) eigenvalue of a symmetric matrix $M \in \mathcal{M}_{n}(\mathbb{R})$ is denoted $\lambda_{\max }(M)$ $\left(\operatorname{resp} . \lambda_{\min }(M)\right) .\lfloor x\rfloor$ denotes the floor of a scalar $x$. $\|$. stands for the Euclidean norm. $\wedge$ defines the "AND", and $\vee$ the "OR" logic gates.

\section{Problem statement}

Consider the perturbed LTI system

$$
\dot{x}(t)=A x(t)+B u(t)+E w(t), \forall t \in \mathbb{R}_{+},
$$

where $x: \mathbb{R}_{+} \rightarrow \mathbb{R}^{n}, u: \mathbb{R}_{+} \rightarrow \mathbb{R}^{n_{u}}$, and $w: \mathbb{R}_{+} \rightarrow \mathbb{R}^{n_{w}}$ represent the state, the control, and the disturbance. $A$, $B$, and $E$ are constant with appropriate dimensions.

The control is a piecewise-constant state feedback

$$
u(t)=-K x\left(t_{k}\right), \forall t \in\left[t_{k}, t_{k+1}\right), \forall k \in \mathbb{N},
$$

with a control gain $K$ such that $A-B K$ is Hurwitz. The sampling instants $t_{k}$ satisfy $t_{0}=0$ and

$$
t_{k+1}-t_{k}=\tau\left(t_{k}, x\left(t_{k}\right)\right) \in\left[\delta, \tau_{\max }\left(x\left(t_{k}\right)\right)\right], \forall k \in \mathbb{N},
$$

with $\delta>0$ ensuring that there is no Zeno phenomenon, a sampling function $\tau: \mathbb{R}_{+} \times \mathbb{R}^{n} \rightarrow \mathbb{R}_{+}$, and a maximal sampling map $\tau_{\max }: \mathbb{R}^{n} \rightarrow \mathbb{R}_{+}$. Here, $\tau_{\max }\left(x\left(t_{k}\right)\right)$ corresponds to "hard" deadline constraints (i.e. a statedependent upper-bound on the sampling intervals), while $\tau\left(t_{k}, x\left(t_{k}\right)\right)$ corresponds to the actual sampling, which may also be subject to uncertainties or jitter. Sufficient conditions for the existence of a sampling map lower-bounded by such a $\delta$ are given in Section 4 .

The disturbance is assumed to be state-bounded, in a similar way as in [35]:

$\exists W \geq 0,\|w(t)\|^{2} \leq W\left\|x\left(t_{k}\right)\right\|^{2}, \forall t \in\left[t_{k}, t_{k+1}\right), \forall k \in \mathbb{N}$.

Such a perturbation can represent model uncertainties or local nonlinearities for example.

We denote by $\mathcal{S}$ the closed-loop system $\{(1),(2),(3),(4)\}$. For given sampling function $\tau$ and disturbance $w$, the solution of $\mathcal{S}$ with initial value $x_{0}=x\left(t_{0}\right)$ is denoted by $x(t)=\varphi_{\tau, w}\left(t, x_{0}\right)$.

Our main objective is to provide a way to enlarge the maximal sampling map $\tau_{\max }$ while ensuring the exponential stability of the system for a given decay-rate $\beta$, also called $\beta$-stability (i.e. such that there exists a scalar $\gamma$ for which all trajectories satisfy $\|x(t)\| \leq \gamma \mathrm{e}^{-\beta t}\left\|x_{0}\right\|$ for any initial condition $\left.x_{0}\right)$. Instead of using a classic Lyapunov approach, which would require to verify that $\dot{V}(t)+2 \beta V(t) \leq 0$ for all trajectories, we propose the following Lyapunov-Razumikhin [19] approach.

Proposition 1 (Adapted from [8]) Consider scalars $\alpha>1, \bar{\sigma}>0,0<\beta \leq \frac{\ln (\alpha)}{2 \bar{\sigma}}, W \geq 0$, and a map $\tau_{\max }: \mathbb{R}^{n} \rightarrow \mathbb{R}_{+}, 0<\delta \leq \tau_{\max }(x) \leq \bar{\sigma}$. If there exists $a$ quadratic function $V(x)=x^{T} P x, P \in \mathbb{S}_{n}^{+*}$ such that

$$
\begin{gathered}
\text { for all } x \in \mathbb{R}^{n}, \text { for all } \sigma \in\left[0, \tau_{\max }(x)\right], \\
\dot{V}\left(\varphi_{\tau_{\max }, w}(\sigma, x)\right)+2 \beta V\left(\varphi_{\tau_{\max }, w}(\sigma, x)\right) \leq 0 \\
\text { whenever } \alpha V\left(\varphi_{\tau_{\max }, w}(\sigma, x)\right) \geq V(x),
\end{gathered}
$$

then the system $\mathcal{S}$ is globally $\beta$-stable.

Before formally formulating the problem under study, let us remark that computing a minimal sampling interval is interesting in practice since it provides an idea 
about the maximal sampling "frequency" the engineer will obtain in the worst case. It is of interest to design the triggering mechanism so as to enlarge this minimal sampling interval. This mechanism strongly depends on the chosen LRF $V$. Therefore, the LRF will be designed so as to enlarge the minimal sampling interval while guaranteeing the expected performance (here, $\beta$-stability). This constitutes the first problem.

\section{Problem 1: Lyapunov function design}

Find a quadratic LRF $V$ such that there exists a sampling map $\tau_{\max }$ satisfying $(\mathrm{C} 1)$ with a minimum value $\tau^{*}=\inf _{x \in \mathbb{R}^{n}} \tau_{\max }(x)$ as large as possible.

Once the LRF $V$ has been obtained, the second problem is the design of the sampling map $\tau_{\max }$ induced by $V$.

\section{Problem 2: Sampling map design}

Given a quadratic LRF $V$, design a sampling map $\tau_{\max }$ such that $(\mathrm{C} 1)$ holds, with $\tau_{\max }(x)$ as large as possible for every $x$.

Problem 1 will be solved in Section 4, while Problem 2 will be solved in Sections 5, 6, and 7, for each dynamic sampling scheme.

\section{$3 \quad$ Main stability results}

In this section, we provide the central stability conditions that will be used for the study of time-varying sampling, event-triggered control, self-triggered control, and statedependent sampling.

Theorem 2 Consider scalars $\alpha>1, \bar{\sigma}>0,0<\beta \leq$ $\frac{\ln (\alpha)}{2 \bar{\sigma}}, W \geq 0$, and a map $\tau_{\max }: \mathbb{R}^{n} \rightarrow \mathbb{R}_{+}, 0<\delta \leq$ $\tau_{\text {max }}(x) \leq \bar{\sigma}$. System $\mathcal{S}$ is globally $\beta$-stable if there exist scalars $\varepsilon \geq 0, \eta \geq 0$, and $\mu \geq 0$, matrices $P, \Psi_{1}, \Psi_{2} \in$ $\mathbb{S}_{n}^{+*}$, and $\bar{\Psi}_{3} \in \mathbb{S}_{n_{w}}^{+*}$, such that

$$
M_{1}+\Psi_{1}+\Psi_{2} \preceq \mu I, \quad\left[\begin{array}{cc}
\Psi_{3}-\eta I & M_{3}^{T} \\
* & -\Psi_{2}
\end{array}\right] \preceq 0,
$$

and

$$
x^{T} \Pi(\sigma) x \leq 0, \forall x \in \mathbb{R}^{n}, \forall \sigma \in\left[0, \tau_{\max }(x)\right],
$$

with

$$
\begin{aligned}
& \Pi(\sigma)=\Lambda(\sigma)^{T} M_{1} \Lambda(\sigma)-\Lambda(\sigma)^{T} P B K-K^{T} B^{T} P \Lambda(\sigma) \\
& -\varepsilon P+M_{2}(\sigma)^{T} \Psi_{1}^{-1} M_{2}(\sigma)+M_{4}(\sigma)^{T} \Psi_{3}^{-1} M_{4}(\sigma) \\
& +W \eta I+\sigma W \mu \lambda_{\max }\left(E^{T} E\right) f_{A}(\sigma) I \\
& M_{1}=A^{T} P+P A+\varepsilon \alpha P+2 \beta P, M_{3}=P E \\
& M_{2}(\sigma)=-P B K+M_{1} \Lambda(\sigma), M_{4}(\sigma)=E^{T} P^{T} \Lambda(\sigma),
\end{aligned}
$$

$$
\begin{gathered}
\Lambda(\sigma)=I+\int_{0}^{\sigma} e^{s A} d s(A-B K), \\
f_{A}(\sigma)=\left\{\begin{array}{c}
\frac{1}{\lambda_{\max }\left(A+A^{T}\right)}\left(e^{\lambda_{\max }\left(A+A^{T}\right) \sigma}-1\right) \\
\text { if } \lambda_{\max }\left(A+A^{T}\right) \neq 0, \\
\sigma \text { otherwise. }
\end{array}\right.
\end{gathered}
$$

The stability conditions in Theorem 2 involve both the sampled state $x\left(t_{k}\right) \triangleq x$ and the time variable $t-t_{k} \triangleq \sigma$. $P$ is the LRF matrix, $\varepsilon$ is a tuning parameter, and the scalars $\eta, \mu$, as well as the matrices $\Psi_{i}$ correspond to degrees of freedom that appear during the majoration of the perturbation terms. In the next section, an algorithm to efficiently compute these parameters will be presented, as a solution to Problem 1.

Remark 1: Following [16], Theorem 2 could be extended to include small delays, by using a transition matrix $\Lambda$ depending on $\sigma$ and the delay. This would lead to triggering conditions depending on both $x\left(t_{k}\right)$ and $x\left(t_{k-1}\right)$. However, this is out of the scope of the paper.

\section{Parameters optimization}

In this section we want to optimize the LRF so as to maximize the lower-bound $\tau^{*}$ of the sampling map $\tau_{\max }$, and thus solve Problem 1.Note that an estimation of the maximal lower-bound $\tau^{*}$ has to verify $\Pi(\sigma) \preceq 0$ for all $\sigma \in\left[0, \tau^{*}\right]$ (see condition (6) in Theorem 2). However, the function $\Pi(\sigma)$ is not a linear expression in the matrices $P$ and $\Psi_{i}, i \in\{1,2,3\}$, and thus the problem is difficult to solve. In the following, we explain how to overcome this issue by transforming the nonlinear constraint into a linear one, to which standard LMI tools/solvers can be applied.

Lemma 3 There exists a sampling map $\tau_{\max }$ lowerbounded by $\tau^{*}$ that satisfies (3), which is such that the condition (6) in Theorem 2 is satisfied if and only if the parameter-dependent $L M I$

$$
\Delta(\sigma)=\left[\begin{array}{ccc}
R(\sigma) & M_{2}(\sigma)^{T} & M_{4}(\sigma)^{T} \\
* & -\Psi_{1} & 0 \\
* & * & -\Psi_{3}
\end{array}\right] \preceq 0
$$

is satisfied for all $\sigma \in\left[0, \tau^{*}\right]$, with

$$
\begin{aligned}
R(\sigma)= & \Lambda(\sigma)^{T} M_{1} \Lambda(\sigma)-\Lambda(\sigma)^{T} P B K-K^{T} B^{T} P \Lambda(\sigma) \\
& -\varepsilon P+W \eta I+\sigma W \mu \lambda_{\max }\left(E^{T} E\right) f_{A}(\sigma) I
\end{aligned}
$$

Lemma 3 requires to check an infinite number of LMI conditions. In order to reduce these conditions to a finite number, we use the following approach. 
Convex embedding according to time: The matrix function $\Delta$ is continuous on the compact set $\left[0, \tau^{*}\right]$. Therefore, it is possible to build a convex polytope defined by a finite set of vertices $\bar{\Delta}_{\kappa}\left(\tau^{*}\right)$, with $\kappa \in \mathcal{K}\left(\tau^{*}\right)$ (a finite set of indexes to be defined later), such that

$$
\begin{gathered}
\left(\bar{\Delta}_{\kappa}\left(\tau^{*}\right) \preceq 0, \forall \kappa \in \mathcal{K}\left(\tau^{*}\right)\right) \\
\Downarrow \\
\left(\Delta(\sigma) \preceq 0, \forall \sigma \in\left[0, \tau^{*}\right]\right) .
\end{gathered}
$$

The shape of the matrix function $\Delta$ in (11) enables to build these vertices as linearly dependent on $P, \Psi_{1}$, $\Psi_{3}, \eta$, and $\mu$, and dependent on the parameters $\alpha, \varepsilon, \beta$, and $\bar{\sigma}$. One possible construction of a convex polytope satisfying (13) is provided in the next subsection.

We are now ready to solve Problem 1.

Theorem 4 Consider $\varepsilon \geq 0$ a tuning parameter. Let scalars $\alpha>1, \bar{\sigma}>0,0<\beta \leq \frac{\ln (\alpha)}{2 \bar{\sigma}}, W \geq 0$, and $0<\delta \leq \tau^{*} \leq \bar{\sigma}$. Let matrices $\bar{\Delta}_{\kappa}\left(\tau^{*}\right)$ satisfying (13), with $\kappa \in \mathcal{K}\left(\tau^{*}\right)$. If there exist matrices $P, \Psi_{1}, \Psi_{2} \in \mathbb{S}_{n}^{+*}$, $\Psi_{3} \in \mathbb{S}_{n_{w}}^{+*}$, and scalars $\eta \geq 0$ and $\mu \geq 0$, such that the LMIs (5) and $\bar{\Delta}_{\kappa}\left(\tau^{*}\right) \preceq 0$ are satisfied for all $\kappa \in \mathcal{K}\left(\tau^{*}\right)$, then there exists a sampling map $\tau_{\max }(x) \geq \tau^{*}, \forall x \in \mathbb{R}^{n}$ such that system $\mathcal{S}$ is globally $\beta$-stable. In particular, system $\mathcal{S}$ with $\delta \leq t_{k+1}-t_{k} \leq \tau^{*}$ for any $k \in \mathbb{N}$ (i.e. with $\left.\tau_{\max }(x)=\tau^{*}, \forall x \in \mathbb{R}^{n}\right)$ is globally $\beta$-stable.

The obtained matrix $P$ corresponds to the LRF which solves Problem 1. It will be used in the following sections to solve Problem 2 while guaranteeing an admissible lower-bound $\tau^{*}$ for the designed sampling maps.

As a side result, it is important to note that Theorem 4 guarantees global $\beta$-stability for arbitrary time-varying sampling $\delta \leq t_{k+1}-t_{k} \leq \tau^{*}$.

Remark 2: The tuning parameter $\varepsilon$ as well as the upperbound $\tau^{*}$ for time-varying sampling can be optimized by using a line-search algorithm and LMI solvers.

\subsection{A convex polytope design}

The conditions from Theorem 4 are based on the use of convex polytopes whose vertices satisfy (13). In this subsection, we propose a convex embedding design satisfying (13) that is adapted from the results in [16]. It is based on a Taylor series approximation of $\Delta$ of order $N$ performed on $l$ subdivision intervals of $[0, \bar{\sigma}]$, of length $\bar{\sigma}$. The idea behind these subdivisions is to design small convex polytopes locally for each time interval subdivision, so as to refine the precision.

Let $\bar{\sigma}>0$, and consider a scalar $0 \leq \tau^{*} \leq \bar{\sigma}$. In this construction, we define the set of vertex indexes

$$
\mathcal{K}\left(\tau^{*}\right)=\{0, \cdots, N\} \times\left\{0, \cdots,\left\lfloor\frac{\tau^{*} l}{\bar{\sigma}}\right\rfloor\right\},
$$

with integers $N \geq 0$ and $l \geq 1$, and design the vertices $\bar{\Delta}_{(i, j)}\left(\tau^{*}\right)$ for all $(i, j) \in \mathcal{K}\left(\bar{\tau}^{*}\right)$, as:

$$
\bar{\Delta}_{(i, j)}\left(\tau^{*}\right)=\hat{\Delta}_{(i, j)}\left(\tau^{*}\right)+\nu I,
$$

with

$$
\hat{\Delta}_{(i, j)}\left(\tau^{*}\right)=\left\{\begin{array}{l}
\sum_{k=0}^{i} \tilde{\Delta}_{(k, j)}\left(\frac{\bar{\sigma}}{l}\right)^{k} \text { if } j<\left\lfloor\frac{\tau^{*} l}{\bar{\sigma}}\right\rfloor, \\
\sum_{k=0}^{i} \tilde{\Delta}_{(k, j)}\left(\tau^{*}-\frac{j \bar{\sigma}}{l}\right)^{k} \text { otherwise }
\end{array}\right.
$$

$$
\begin{gathered}
\tilde{\Delta}_{(0, j)}=\left[\begin{array}{ccc}
L_{0, j}-K^{T} B^{T} P+\Gamma_{1, j}^{T} M_{1}^{T} & \Gamma_{1, j}^{T} P E \\
* & -\Psi_{1} & 0 \\
* & * & -\Psi_{3}
\end{array}\right], \\
\tilde{\Delta}_{(k \geq 1, j)}=\left[\begin{array}{ccc}
L_{k, j} & \Gamma_{2, j}^{T} \frac{\left(A^{k-1}\right)^{T}}{k !} M_{1}^{T} & \Gamma_{2, j}^{T} \frac{\left(A^{k-1}\right)^{T}}{k !} P E \\
* & 0 & 0 \\
* & * & 0
\end{array}\right],
\end{gathered}
$$

$$
\begin{aligned}
\Gamma_{1, j}= & I+N_{j}(A-B K), \Gamma_{2, j}=N_{j}^{\prime}(A-B K), \\
N_{j}= & \int_{0}^{j \bar{\sigma}} \mathrm{e}^{A s} d s, N_{j}^{\prime}=A N_{j}+I, \\
L_{0, j}= & \Gamma_{1, j}^{T} M_{1} \Gamma_{1, j}-\varepsilon P+W \eta I \\
& \quad-\Gamma_{1, j}^{T} P B K-K^{T} B^{T} P \Gamma_{1, j}+\tilde{L}_{0, j}, \\
L_{1, j}= & \Gamma_{2, j}^{T}\left(M_{1} \Gamma_{1, j}-P B K\right) \\
& +\left(\Gamma_{1, j}^{T} M_{1}^{T}-K^{T} B^{T} P\right) \Gamma_{2, j}+\tilde{L}_{1, j}, \\
L_{k \geq 2, j}= & \Gamma_{2, j}^{T} \frac{\left(A^{k-1}\right)^{T}}{k !}\left(M_{1} \Gamma_{1, j}-P B K\right) \\
& \quad+\left(\Gamma_{1, j}^{T} M_{1}^{T}-K^{T} B^{T} P\right) \frac{A^{k-1}}{k !} \Gamma_{2, j} \\
& \quad+\Gamma_{2, j}^{T}\left(\sum_{i=1}^{k-1} \frac{\left(A^{i-1}\right)^{T}}{i !} M_{1} \frac{A^{k-i-1}}{(k-i) !}\right) \Gamma_{2, j}+\tilde{L}_{k, j} .
\end{aligned}
$$

If $\lambda_{\max }\left(A+A^{T}\right)=0$, the matrices $\tilde{L}_{k, j}$ are defined as

$$
\begin{aligned}
& \tilde{L}_{0, j}=W \mu \lambda_{\max }\left(E^{T} E\right)\left(j \frac{\bar{\sigma}}{l}\right)^{2} I, \\
& \tilde{L}_{1, j}=2 W \mu \lambda_{\max }\left(E^{T} E\right) j \frac{\bar{\sigma}}{l} I, \\
& \tilde{L}_{2, j}=W \mu \lambda_{\max }\left(E^{T} E\right) I, \\
& \tilde{L}_{k \geq 3, j}=0 .
\end{aligned}
$$

Otherwise, if $\lambda_{\max }\left(A+A^{T}\right) \neq 0$, they are defined as

$$
\begin{aligned}
\tilde{L}_{0, j}= & W \mu \frac{\lambda_{\max }\left(E^{T} E\right)}{\lambda_{\max }\left(A+A^{T}\right)} j \frac{\bar{\sigma}}{l}\left(\mathrm{e}^{\lambda_{\max }\left(A+A^{T}\right) j \frac{\bar{\sigma}}{l}}-1\right) I, \\
\tilde{L}_{1, j}= & W \mu \frac{\lambda_{\max }\left(E^{T} E\right)}{\lambda_{\max }\left(A+A^{T}\right)} \\
& \left(\mathrm{e}^{\lambda_{\max }\left(A+A^{T}\right) j \bar{\sigma}}\left(1+j \frac{\bar{\sigma}}{l} \lambda_{\max }\left(A+A^{T}\right)\right)-1\right) I,
\end{aligned}
$$




$$
\begin{aligned}
\tilde{L}_{k \geq 2, j}= & W \mu \frac{\lambda_{\max }\left(E^{T} E\right)}{\lambda_{\max }\left(A+A^{T}\right)} \mathrm{e}^{\lambda_{\max }\left(A+A^{T}\right) j \overline{\bar{\sigma}}} \\
& \left(j \frac{\bar{\sigma}}{l} \frac{\left(\lambda_{\max }\left(A+A^{T}\right)\right)^{k}}{k !}+\frac{\left(\lambda_{\max }\left(A+A^{T}\right)\right)^{k-1}}{(k-1) !}\right) I .
\end{aligned}
$$

Finally,

$$
\nu \geq \max _{\substack{\sigma^{\prime} \in[0, \overline{\bar{\sigma}}], r \in\{0, \cdots, l-1\}}} \lambda_{\max }\left(\Delta\left(\sigma^{\prime}+r \frac{\bar{\sigma}}{l}\right)-\sum_{k=0}^{N} \tilde{\Delta}_{(k, r)} \sigma^{\prime k}\right) .
$$

Remark 3: The matrices $\tilde{\Delta}_{(k, j)}$ defined in (17) are the coefficients of the Taylor polynomial of $\Delta$. Indeed, it can be shown (see the proof of Lemma 5) that the polynomial approximation of $\Delta$ of order $N$ for $\sigma \in\left[j \frac{\bar{\sigma}}{l},(j+1) \frac{\bar{\sigma}}{l}\right]$, with $j \in\{0, \cdots, l-1\}$, is expressed as $\sum_{k=0}^{N} \tilde{\Delta}_{(k, j)}\left(\sigma-j \frac{\bar{\sigma}}{l}\right)^{k}$.

The constant $\nu$ defined in (22) represents an upperbound of the Taylor series approximation error: it can be shown (see the proof of Lemma 5) that $\Delta(\sigma)-\sum_{k=0}^{N} \tilde{\Delta}_{(k, j)}\left(\sigma-j \frac{\bar{\sigma}}{l}\right)^{k} \preceq \nu I$ for all $\sigma \in$ $\left[j \frac{\bar{\sigma}}{l},(j+1) \frac{\bar{\sigma}}{l}\right]$ and $j \in\{0, \cdots, l-1\}$.

Lemma 5 Consider a scalar $\tau^{*} \in[0, \bar{\sigma}]$. The vertices $\bar{\Delta}_{(i, j)}\left(\tau^{*}\right)$ with indexes $\mathcal{K}\left(\tau^{*}\right)$ defined in (15) and (14) satisfy the property (13): if the condition $\bar{\Delta}_{(i, j)}\left(\tau^{*}\right) \preceq 0$ is satisfied for all $(i, j) \in \mathcal{K}\left(\tau^{*}\right)$, then $\Delta(\sigma) \preceq 0$ for all $\sigma \in\left[0, \tau^{*}\right]$.

Lemma 5 is a key to solve Problem 1 in a numerical way.

\subsection{Optimization algorithm based on the proposed poly- tope design}

Below is provided an algorithm that allows for computing an estimation of the maximal $\tau^{*}$ such that there exist a sampling map that stabilizes the system according to Theorem 4 with the proposed convex polytope design, and which satisfies $\tau_{\max }(x) \geq \tau^{*}, \forall x \in \mathbb{R}^{n}$.

Step 1: Use Theorem 4 and the polytopic description (15) considering an upper-bound on the estimation error $\nu=0$. The search for $P, \Phi_{1}, \Phi_{2}, \Phi_{3}, \eta$ and $\mu$ is then an LMI problem, and we may compute an estimation $\hat{\tau}^{*}$ of the largest sampling interval $\tau^{*}$ satisfying the conditions from Theorem 4, as well as its associated parameter $\varepsilon$, by using a line-search algorithm, as proposed in Remark 2 . Step 2: Compute the upper-bound $\nu$ corresponding to the obtained parameters $P, \varepsilon, \Phi_{1}, \Phi_{2}, \Phi_{3}, \eta$ and $\mu$, following (22). Then, it is possible to evaluate the matrix inequalities $\bar{\Delta}_{\kappa}\left(\tau^{*}\right) \preceq 0$ in Theorem 4 so as to obtain an estimation of the largest upper-bound $\tau^{*}$ for timevarying samplings satisfying the stability conditions.

Remark 4: Using the LRF $V(x)=x^{T} P x$ together with $\varepsilon, \Psi_{1}, \Psi_{2}, \Psi_{3}, \eta, \mu$ and $\nu$ obtained thanks to this algorithm allows for designing sampling rules that are lower- bounded by $\tau^{*}$ in the case of event-triggered control, self-triggered control, and state-dependent sampling.

\section{Event-triggered control}

In this section, we solve Problem 2 using an eventtriggered control scheme based on Theorem 2. To begin with, it is clear that system $\mathcal{S}$ is $\beta$-stable with an eventgenerator for the $(k+1)^{\text {th }}$ sampling defined as

$$
\left(x\left(t_{k}\right)^{T} \Pi\left(t-t_{k}\right) x\left(t_{k}\right)=0\right) \vee\left(t=t_{k}+\bar{\sigma}\right),
$$

with matrix $\Pi\left(t-t_{k}\right)$ defined in (7), and parameters $P, \varepsilon, \Psi_{1}, \Psi_{2}, \Psi_{3}, \eta$, and $\mu$ satisfying the conditions from Theorem 2. Furthermore, if we use parameters that satisfy the conditions of Theorem 4 (for example those obtained using the algorithm in Subsection 4.2, which maximizes the lower-bound $\tau^{*}$ of the sampling map), then there is no need to check the triggering condition (23) during the time $t \in\left[t_{k}, t_{k}+\tau^{*}\right]$ since Theorem 4 ensures $x\left(t_{k}\right)^{T} \Pi\left(t-t_{k}\right) x\left(t_{k}\right) \leq 0$ for all $t \in\left[t_{k}, t_{k}+\tau^{*}\right]$.

Proposition 6 Consider parameters $P, \varepsilon, \Psi_{1}, \Psi_{2}, \Psi_{3}$, $\eta, \mu$, and $\tau^{*}$ satisfying the conditions from Theorem 4 . Then, system $\{(1),(2),(4)\}$ with the triggering condition

$$
\left(t \geq t_{k}+\tau^{*}\right) \wedge\left(\left(x\left(t_{k}\right)^{T} \Pi\left(t-t_{k}\right) x\left(t_{k}\right)=0\right) \vee\left(t=t_{k}+\bar{\sigma}\right)\right),
$$

for $k \in \mathbb{N}$, is globally $\beta$-stable. This event-trigger generates a sampling sequence $t_{k+1}-t_{k}=\tau_{\max }\left(x\left(t_{k}\right)\right)$, where

$$
\tau_{\max }(x)=\min \left(\min \left\{\sigma \geq \tau^{*} \mid x^{T} \Pi(\sigma) x=0\right\}, \bar{\sigma}\right) .
$$

This scheme will be used as a referential for a comparison, in order to check the conservatism introduced in the self-triggered and the state-dependent sampling schemes presented in the next sections.

\section{Self-triggered control}

In this section, we solve Problem 2 using a self-triggered control scheme. We use Theorem 2 and convexification arguments (13) for that purpose. For a given sampling interval $\tilde{\sigma} \in[0, \bar{\sigma}]$, we want to characterize the regions of the state space for which the stability conditions (6) from Theorem 2 (i.e. the decay of the LRF) is satisfied for all $\sigma \in[0, \tilde{\sigma}]$ :

$$
\mathcal{R}(\tilde{\sigma})=\left\{x \in \mathbb{R}^{n} \mid x^{T} \Pi(\sigma) x \leq 0, \forall \sigma \in[0, \tilde{\sigma}]\right\} .
$$

This set is defined as the intersection of an infinite number of conic regions, and is therefore difficult to compute a priori. The idea we propose is to under-approximate $\mathcal{R}(\tilde{\sigma})$ by a finite number of conic regions

$$
\hat{\mathcal{R}}(\tilde{\sigma})=\left\{x \in \mathbb{R}^{n} \mid x^{T} \Pi_{\kappa}(\tilde{\sigma}) x \leq 0, \forall \kappa \in \mathcal{K}(\tilde{\sigma})\right\},
$$


with a finite number of matrices $\Pi_{\kappa}(\tilde{\sigma})$ and a finite set of indexes $\kappa \in \mathcal{K}(\tilde{\sigma})$, designed such that $\hat{\mathcal{R}}(\tilde{\sigma}) \subseteq \mathcal{R}(\tilde{\sigma})$.

Theorem 7 Consider a subset of sampling intervals $\Upsilon \subset[0, \bar{\sigma}]$, scalars $\alpha>1, \bar{\sigma}>0,0<\beta \leq \frac{\ln (\alpha)}{2 \bar{\sigma}}, W \geq 0$, $\varepsilon \geq 0, \eta \geq 0, \mu \geq 0$, and matrices $P, \Psi_{1}, \Psi_{2} \in \mathbb{S}_{n}^{+*}$, $\Psi_{3} \in \mathbb{S}_{n_{w}}^{+*}$, such that the LMIs (5) hold.

Assume that for any $\tilde{\sigma} \in[0, \bar{\sigma}]$, there exist matrices $\Pi_{\kappa}(\tilde{\sigma}) \in \mathcal{M}_{n}(\mathbb{R})$, with $\kappa \in \mathcal{K}(\tilde{\sigma})$ a finite set of indexes such that $\hat{\mathcal{R}}(\tilde{\sigma}) \subseteq \mathcal{R}(\tilde{\sigma})$, with the sets $\mathcal{R}(\tilde{\sigma})$ and $\hat{\mathcal{R}}(\tilde{\sigma})$ defined in (24) and (25) respectively. Then, the system $\mathcal{S}$ is globally $\beta$-stable with the sampling map

$$
\tau_{\max }(x)=\underset{\tilde{\sigma} \in \Upsilon}{\arg \max }\{x \in \hat{\mathcal{R}}(\tilde{\sigma})\} .
$$

Remark 5: From condition (24), it is clear that for $\tilde{\sigma}_{1}<$ $\tilde{\sigma}_{2}$, we have $\mathcal{R}\left(\tilde{\sigma}_{1}\right) \subset \mathcal{R}\left(\tilde{\sigma}_{2}\right)$. In order to reduce the online computational load, it is worth considering regions $\hat{\mathcal{R}}(\tilde{\sigma})$ such that for $\tilde{\sigma}_{1}<\tilde{\sigma}_{2}$, we have both $\hat{\mathcal{R}}\left(\tilde{\sigma}_{1}\right) \subset \hat{\mathcal{R}}\left(\tilde{\sigma}_{2}\right)$, $\mathcal{K}\left(\tilde{\sigma}_{1}\right) \subset \mathcal{K}\left(\tilde{\sigma}_{2}\right)$, and $\Pi_{\kappa}\left(\tilde{\sigma}_{1}\right)=\Pi_{\kappa}\left(\tilde{\sigma}_{2}\right)=\Pi_{\kappa}$, for all $\kappa \in \mathcal{K}\left(\tilde{\sigma}_{1}\right)$. The main advantage to such considerations is that the matrices $\Pi_{\kappa}$ do not depend on $\tilde{\sigma}$, and can thus be computed offline, for all $\kappa \in \mathcal{K}(\bar{\sigma})$, once for all.

\subsection{Self-triggered control scheme based on the proposed polytope design}

We can now design numerically the regions (25) thanks to the polytopic construction in Subsection 4.1.

Step 1: To maximize the lower-bound $\tau^{*}$ of the sampling map, consider the parameters $P, \varepsilon, \Phi_{1}, \Phi_{2}, \Phi_{3}, \eta, \mu$ and $\nu$ obtained using the method proposed in Section 4.

Step 2: Consider a grid of sampling intervals $\Upsilon=$ $\left.\overline{\left\{\sigma_{1}, \cdots\right.}, \sigma_{l}\right\}$, with $\sigma_{i}=\frac{i}{l} \bar{\sigma}$. Here, $l$ corresponds to both the number of sampling intervals considered in the grid, and the number of polytopic subdivisions from the convex embedding design (see Subsection 4.1).

Step 3: Using the polytopic design (14), (15), compute the matrices $\bar{\Delta}_{\kappa}(\bar{\sigma})$, with $\kappa \in \mathcal{K}(\bar{\sigma})$. They take the form

$$
\bar{\Delta}_{\kappa}(\bar{\sigma})=\left[\begin{array}{ccc}
\Delta_{\kappa}^{(1,1)} & \Delta_{\kappa}^{(1,2)} & \Delta_{\kappa}^{(1,3)} \\
* & \Delta_{\kappa}^{(2,2)} & 0 \\
* & * & \Delta_{\kappa}^{(3,3)}
\end{array}\right] .
$$

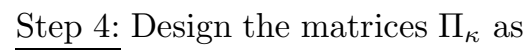

$$
\begin{aligned}
\Pi_{\kappa}= & \Delta_{\kappa}^{(1,1)}-\Delta_{\kappa}^{(1,2)}\left[\Delta_{\kappa}^{(2,2)}\right]^{-1}\left[\Delta_{\kappa}^{(1,2)}\right]^{T} \\
& -\Delta_{\kappa}^{(1,3)}\left[\Delta_{\kappa}^{(3,3)}\right]^{-1}\left[\Delta_{\kappa}^{(1,3)}\right]^{T}
\end{aligned}
$$

and the set of indexes $\mathcal{K}\left(\sigma_{i}\right)$ as in (14). For $\sigma_{i} \in \Upsilon$, with $\Upsilon$ designed in Step 2, we get

$$
\mathcal{K}\left(\sigma_{i}\right)=\{0, \cdots, N\} \times\{0, \cdots, i-1\},
$$

Step 5: Taking advantage of Step 1 , since $\tau^{*}$ is designed such that $\mathcal{R}\left(\tau^{*}\right)=\mathbb{R}^{n}$, the sampling map (26) yields

$$
\tau_{\max }(x)=\tau_{\max }^{\Upsilon}(x)=\max \left(\underset{\tilde{\sigma} \in \Upsilon}{\arg \max }\{x \in \hat{\mathcal{R}}(\tilde{\sigma})\}, \tau^{*}\right) .
$$

The matrices $\Pi_{\kappa}$ in (28) come from the Schur complement of the matrices $\vec{\Delta}_{\kappa}(\bar{\sigma})$ in $(27)$. As in Lemma 5 , where it was proven that (13) holds with vertices (15), we can show that with the matrices $\Pi_{\kappa}$ defined in (28), and the set of indexes $\mathcal{K}\left(\sigma_{i}\right)(29)$, the set $\hat{\mathcal{R}}(\tilde{\sigma})(25)$ satisfies $\hat{\mathcal{R}}(\tilde{\sigma}) \subseteq \mathcal{R}(\tilde{\sigma})$.

Remark 6: With this construction and the parameters considered in Step 1, Theorem 4 ensures that if $\left\lfloor\frac{\tau^{*} l}{\bar{\sigma}}\right\rfloor \geq 1$, then $\Pi_{(i, j)} \preceq 0$ for all $(i, j) \in\{0, \cdots, N\} \times$ $\left\{0, \cdots,\left\lfloor\frac{\tau^{*} l}{\bar{\sigma}}\right\rfloor-1\right\}$. Therefore, when computing online $\tau_{\max }(x)$, using (30) and (25), it is not necessary to check the inequalities $x^{T} \Pi_{(i, j)} x \leq 0$ for $j<\left\lfloor\frac{\tau^{*} l}{\bar{\sigma}}\right\rfloor$.

Remark 7: The precision of the sampling map $\tau_{\max }$ is linked to the precision of the grid $\Upsilon$. In our construction, this corresponds to the integer $l$, which defines the number subdivisions of the time interval $[0, \bar{\sigma}]$ used in the construction of the convex polytope (15). The larger the $l$, the more precise the sampling map (30).

The number of online computations required to compute the next maximal allowable sampling interval $\tau_{\max }$ using (30) is upper-bounded by $n(n+1)(N+1)\left(l-\left\lfloor\frac{\tau^{*} l}{\bar{\sigma}}\right\rfloor\right)$ multiplications and $(n+1)(n-1)(N+1)\left(l-\left\lfloor\frac{\tau^{*} l}{\bar{\sigma}}\right\rfloor\right)$ additions. The online complexity is $O\left(N \ln { }^{2}\right)$. It is comparable to the one obtained in the self-triggered control scheme from [25] for example. Here some computations are saved thanks to the optimization of the lower-bound $\tau^{*}$ of the sampling map (Step 1, see Remark 6).

\section{State-dependent sampling}

In this section, we solve Problem 2 using a statedependent sampling scheme. In this formulation, the sampling map is defined over regions of the state-space:

$$
\tau_{\max }(x)=\tau_{\max }^{(s)}, \forall x \in \mathcal{R}_{s}, \forall s \in\{1, \cdots, q\} .
$$

For any state $x \neq 0$, the condition (6) from Theorem 2 remains the same for any state $y=\lambda x, \lambda \in \mathbb{R}^{*}$. Therefore, it is sufficient to work with homogeneous sampling maps of degree 0 (i.e. satisfying $\tau_{\max }(\lambda x)=\tau_{\max }(x)$ for all $x \in \mathbb{R}^{n}, \lambda \in \mathbb{R}^{*}$ ) and to check condition (6) over the unit $n$-sphere, as in [1] and [8]. This motivates us for working with conic regions of the form

$$
\mathcal{R}_{s}=\left\{x \in \mathbb{R}^{n}, x^{T} \Phi_{s} x \geq 0\right\}, \Phi_{s}=\Phi_{s}^{T} \in \mathcal{M}_{n}(\mathbb{R}) .
$$

Possible constructions of these conic regions are presented in [8], using the spherical coordinates (isotropic 
covering) or the discrete-time behaviour of the system (anisotropic covering). We have the following property.

Theorem 8 Consider scalars $\alpha>1, \bar{\sigma}>0,0<\beta \leq$ $\frac{\ln (\alpha)}{2 \bar{\sigma}}, W \geq 0, \varepsilon \geq 0, \eta \geq 0, \mu \geq 0$, and matrices $P, \Psi_{1}, \Psi_{2} \in \mathbb{S}_{n}^{+*}, \Psi_{3} \in \mathbb{S}_{n_{w}+\text { }}^{+*}$ such that the LMIs (5) hold. Consider the sampling map (31) defined on conic regions (32), with sampling intervals $\tau_{\max }^{(1)}, \cdots, \tau_{\max }^{(q)}$ satisfying $0<\delta \leq \tau_{\max }^{(s)} \leq \bar{\sigma}$. Assume there exist matrices $\bar{\Delta}_{\kappa}\left(\tau_{\max }^{(s)}\right)$, with $\kappa \in \mathcal{K}\left(\tau_{\max }^{(s)}\right)$ a finite set of indexes, satisfying for all $s \in\{1, \cdots, q\}$, and $\rho_{s} \geq 0$,

$$
\begin{gathered}
\left(\bar{\Delta}_{\kappa}\left(\tau_{\text {max }}^{(s)}\right)+\left[\begin{array}{cr}
\rho_{s} \Phi_{s} & 0 \\
* & 0
\end{array}\right] \preceq 0, \forall \kappa \in \mathcal{K}\left(\tau_{\text {max }}^{(s)}\right)\right) \\
\Downarrow \\
\left(\Delta(\sigma)+\left[\begin{array}{cc}
\rho_{s} \Phi_{s} & 0 \\
* & 0
\end{array}\right] \preceq 0, \forall \sigma \in\left[0, \tau_{\text {max }}^{(s)}\right]\right) .
\end{gathered}
$$

Then, if there exist scalars $\rho_{s} \geq 0$ such that the LMI conditions (33a) are satisfied for all $s \in\{1, \cdots, q\}$, the system $\mathcal{S}$ with the sampling map $\tau_{\max }$ is globally $\beta$-stable.

\subsection{State-dependent sampling scheme based on the pro- posed polytope design}

A possible design of matrices $\bar{\Delta}_{\kappa}\left(\tau_{\max }^{(s)}\right), \kappa \in \mathcal{K}\left(\tau_{\max }^{(s)}\right)$, is proposed in Subsection 4.1. Indeed, by adapting the proof of Lemma 5 , one can show that these matrices satisfy (33) for all $s \in\{1, \cdots, q\}$ and any $\rho_{s} \geq 0$.

A method to compute a lower-bound of the optimal sampling map, solution of Problem 2, is proposed.

Step 1: In order to maximize the lower-bound $\tau^{*}$ of the sampling map, consider the parameters $P, \varepsilon, \Phi_{1}, \Phi_{2}, \Phi_{3}$, $\eta, \mu$ and $\nu$ obtained using the method proposed in Section 4.

Step 2: Use the LMI conditions from Theorem 8 in order to maximize the sampling intervals $\tau_{\max }^{(s)}$ on each region, using a line search algorithm.

Step 3: Design a lower-bound estimation of the optimal sampling map $\tau_{\text {opt }}^{V}$ as proposed in (31):

$$
\tau_{\max }(x)=\tau_{\max }^{(s)}, \forall x \in \mathcal{R}_{s}, s \in\{1, \cdots, q\} .
$$

Remark 8: The online complexity of the statedependent sampling approach depends on the design of the conic covering. With the anisotropic covering proposed in [8], the online complexity is $O\left(q n^{2}\right)$, and it can be shown that for the same precision, the number of computations in that case is divided by $N$ compared to the self-triggered control case (Section 6). With the isotropic covering proposed in [8], the online complexity becomes $O(n)$. Additionally, in that latter case, the online complexity does not depend on the number of regions (i.e. on the precision).

\begin{tabular}{|c|c||c|c|}
\hline Method & $\tau^{*}$ & Method & $\tau^{*}$ \\
\hline$[13],[20]$ & $0.3347 s$ & {$[32]$} & $0.5200 s$ \\
\hline$[11],[21]$ & $0.4244 s$ & {$[4]$} & $0.5376 s$ \\
\hline$[9]($ LKF $)$ & $0.4305 s$ & Theorem 4 & $0.5421 s$ \\
\hline$[7]$ (LF+embed.) & $0.4578 s$ & {$[8]$ (LRF+embed.) } & $0.5938 s$ \\
\hline
\end{tabular}

Table 1

Maximum upper-bounds $\tau^{*}$ for time-varying sampling, without perturbations nor guaranteed decay-rate

\section{Numerical example}

Consider the system from [33]:

$\dot{x}(t)=\left[\begin{array}{cc}0 & 1 \\ -2 & 3\end{array}\right] x(t)-\left[\begin{array}{l}0 \\ 1\end{array}\right] K x\left(t_{k}\right)+w(t), K=\left[\begin{array}{ll}-1 & 4\end{array}\right]$.

In the following, we set the polynomial approximation degree term $N=5$ and the number of polytopic subdivisions $l=100$. For a given $\beta$, after fixing $\bar{\sigma}$, we set the LRF performance parameter $\alpha>1$ (see Proposition 1) as small as possible and such that $\beta \leq \frac{\ln (\alpha)}{2 \bar{\sigma}}$.

Stability analysis for time-varying sampling: We use the algorithm proposed in Section 4 to perform a robust stability analysis with respect to time-varying sampling for given values of parameters $\beta$ and $W$. The table in Figure 1 shows the upper-bounds $\tau^{*}$ for time-varying sampling obtained for $\beta=0.1$ and different values of $W$, while Table 1 presents a comparison with some upperbounds from the literature, thus limited to the unperturbed case, without decay-rate. These bounds are close to the practical upper-bound obtained for periodic sampling: $T_{\text {Schur }}=0.5948 \mathrm{~s}$.

State-dependent sampling map: We set $q=100$ conic regions (isotropic partition on the unit sphere $x=\mathrm{e}^{\mathrm{i} \theta}$, $\theta \in[-\pi, \pi]$, see the design in [8]), and use the method in Section 7 to design the maximal sampling maps for given parameters $\beta$ and $W$. Figure 1 shows results for $\beta=0.1$. Dynamic sampling control: In Figure 2, we present the inter-execution times obtained in simulations for $\beta=$ 0.1 and $W=0.04$ (i.e. with a perturbation $\|w(t)\| \leq$ $\left.20 \%\left\|x\left(t_{k}\right)\right\|\right)$, and initial condition $x_{0}=\left[\begin{array}{ll}5 & 3\end{array}\right]^{T}$, with event-triggered control, self-triggered control, and statedependent sampling. The similar evolutions illustrate the low conservatism introduced by the convex embedding (13) (used in the self-triggered control and the statedependent sampling schemes) and the conic regions (32) (used in the state-dependent sampling scheme).

\section{Conclusion}

We have introduced a Lyapunov-Razumikhin-based design of a sampling map in the state space. Respecting this map $\tau_{\text {max }}$ ensures the exponential stability with a given decay-rate for perturbed linear state feedback systems. The proposed method can be used to perform ro- 


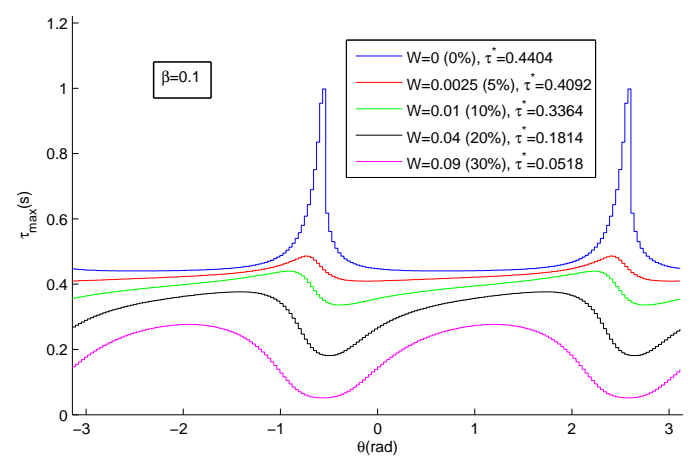

Fig. 1. State-angle-dependent sampling map $\tau_{\max }$ for a decay-rate $\beta=0.1$ and different values of $W$

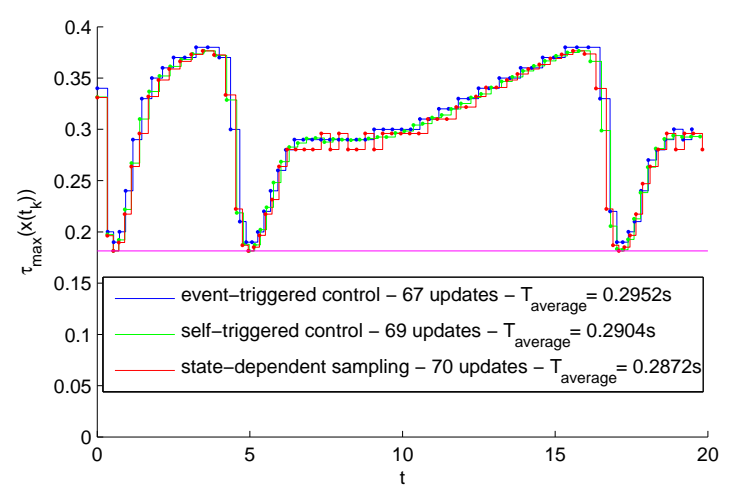

Fig. 2. Inter-execution times $\tau_{\max }\left(x\left(t_{k}\right)\right)$ for a decay rate $\beta=0.1$ and $W=0.04\left(\|w(t)\| \leq 20 \%\left\|x\left(t_{k}\right)\right\|\right)$

bust stability analysis with respect to time-varying sampling, event-triggered control, self-triggered control, and state-dependent sampling. A lower-bound estimation of the maximal sampling map is proposed for all of these approaches. The method presents several advantages:

- It makes it possible to maximize the lower-bound $\tau^{*}$ of the proposed map, whatever the sampling technique.

- It provides the associated LRF parameters.

- The map of the next maximal sampling interval with respect to the past sampled state value can be designed offline (state-dependent sampling).

Extensions to sampled-data systems with small delays and to other dynamic sampling control schemes (periodic event-triggered control) are currently under study.

\section{Appendix}

Lemma 9 Consider scalars $\alpha>1, \bar{\sigma}>0,0<\beta \leq$ $\frac{\ln (\alpha)}{2 \bar{\sigma}}$, and $W \geq 0$, and a map $\tau_{\max }: \mathbb{R}^{n} \rightarrow \mathbb{R}_{+}, 0<\delta \leq$ $\tau_{\text {max }}^{2 \sigma}(x) \leq \bar{\sigma}$. Then, the system $\mathcal{S}$ is globally $\beta$-stable if there exist a matrix $P \in \mathbb{S}_{n}^{+*}$ and a scalar $\varepsilon \geq 0$ such that for all $x \in \mathbb{R}^{n}$, and all $\sigma \in\left[0, \tau_{\max }(x)\right]$,

$$
\chi_{\omega}(\sigma, x)^{T} \Omega \chi_{\omega}(\sigma, x) \leq 0,
$$

$$
\begin{gathered}
\text { with } \chi_{\omega}(\sigma, x)=\left[\Lambda(\sigma) x+J_{w}(\sigma) x w(\sigma)\right]^{T}, \\
\Omega=\left[\begin{array}{ccc}
A^{T} P+P A+\varepsilon \alpha P+2 \beta P & -P B K & P E \\
* & -\varepsilon P & 0 \\
* & * & 0
\end{array}\right], \\
J_{w}(\sigma)=\int_{0}^{\sigma} e^{A(\sigma-s)} E w(s) d s,
\end{gathered}
$$

and $\Lambda(\sigma)$ designed in $(9)$.

Proof: Using the dynamics of $\mathcal{S}$, we rewrite $\alpha V\left(\varphi_{\tau_{\max }, w}(\sigma, x)\right) \geq V(x)$ from $(\mathrm{C} 1)$ as $\left[\begin{array}{c}\varphi_{\tau_{\max }, w}(\sigma, x) \\ x\end{array}\right]^{T}\left[\begin{array}{cc}-\alpha P & 0 \\ * & P\end{array}\right]\left[\begin{array}{c}\varphi_{\tau_{\max }, w}(\sigma, x) \\ x\end{array}\right] \leq 0$, and $\dot{V}\left(\varphi_{\tau_{\max }, w}(\sigma, x)\right)+2 \beta V\left(\varphi_{\tau_{\max }, w}(\sigma, x)\right) \leq 0$ as $\tilde{\chi}_{\omega}(\sigma, x)^{T} \tilde{\Omega} \tilde{\chi}_{\omega}(\sigma, x) \leq 0$, with $\tilde{\Omega}=\left.\Omega\right|_{\varepsilon=0}$ and $\tilde{\chi}_{\omega}(\sigma, x)=$ $\left[\varphi_{\tau_{\max }, w}(\sigma, x) x w(\sigma)\right]^{T}$. Noting that the evolution of the state satisfies $\varphi_{\tau_{\max }, w}(\sigma, x)=\Lambda(\sigma) x+J_{w}(\sigma)$ and using the S-procedure shows that the conditions from Proposition 1 hold if the ones from Lemma 9 hold.

Proof of Proposition 1: The proof is essentially the same as the one in the unperturbed case in [8]. The existence and uniqueness of solutions in the perturbed case can be shown as in the framework [24], using the locally essentially boundedness of the perturbation $w$.

Proof of Theorem 2: We want to find an upper-bound independent of the perturbation $w$ for the left part of equation (34), which is equal to

$$
\begin{aligned}
& G_{w}(\sigma, x)= \\
& x^{T}\left(\Lambda(\sigma)^{T} M_{1} \Lambda(\sigma)-\Lambda(\sigma)^{T} P B K-K^{T} B^{T} P \Lambda(\sigma)-\varepsilon P\right) x \\
& +w(\sigma)^{T} M_{4}(\sigma) x+x^{T} M_{4}(\sigma)^{T} w(\sigma) \\
& +J_{w}(\sigma)^{T} M_{2}(\sigma) x+x^{T} M_{2}(\sigma)^{T} J_{w}(\sigma) \\
& +J_{w}(\sigma)^{T} M_{3} w(\sigma)+w(\sigma)^{T} M_{3}^{T} J_{w}(\sigma)+J_{w}(\sigma)^{T} M_{1} J_{w}(\sigma) .
\end{aligned}
$$

To do so, we use the inequality in [14], Lemma 6.2, to show that for any matrices $\Psi_{1}, \Psi_{2} \in \mathbb{S}_{n}^{+*}$, and $\Psi_{3} \in \mathbb{S}_{n_{w}^{+*}}$,

$$
\begin{aligned}
& G_{w}(\sigma, x) \leq w(\sigma)^{T}\left[M_{3}^{T} \Psi_{2}^{-1} M_{3}+\Psi_{3}\right] w(\sigma) \\
& \quad+J_{w}(\sigma)^{T}\left[M_{1}+\Psi_{1}+\Psi_{2}\right] J_{w}(\sigma) \\
& +x^{T}\left[\Lambda(\sigma)^{T} M_{1} \Lambda(\sigma)-\Lambda(\sigma)^{T} P B K-K^{T} B^{T} P \Lambda(\sigma)\right. \\
& \left.\quad-\varepsilon P+M_{2}(\sigma)^{T} \Psi_{1}^{-1} M_{2}(\sigma)+M_{4}(\sigma)^{T} \Psi_{3}^{-1} M_{4}(\sigma)\right] x
\end{aligned}
$$

Using assumption (4), we upper-bound the term $w(\sigma)^{T}\left[M_{3}^{T} \Psi_{2}^{-1} M_{3}+\Psi_{3}\right] w(\sigma)$ in (37) by $W \lambda_{\max }\left(M_{3}^{T} \Psi_{2}^{-1} M_{3}+\Psi_{3}\right) x^{T} x$. 
Then, with a scalar $\eta \geq 0$ such that $\left[\begin{array}{cc}\Psi_{3}-\eta I & M_{3}^{T} \\ * & -\Psi_{2}\end{array}\right] \preceq$ 0 , as assumed in (5), and using the Schur complement, one obtains

$$
w(\sigma)^{T}\left[M_{3}^{T} \Psi_{2}^{-1} M_{3}+\Psi_{3}\right] w(\sigma) \leq W \eta x^{T} x
$$

Let us denote $Q=M_{1}+\Psi_{1}+\Psi_{2}$. Using (5), we upperbound the term $J_{w}(\sigma)^{T} Q J_{w}(\sigma)$ in (37) by $\mu\left(\int_{0}^{\sigma} \mathrm{e}^{A(\sigma-s)} E w(s) d s\right)^{T}\left(\int_{0}^{\sigma} \mathrm{e}^{A(\sigma-s)} E w(s) d s\right)$. Then, using Jensen's inequality, the inequality (2.2) in [22], along with classic inequalities and assumption (4), one gets

$$
\begin{aligned}
& J_{w}(\sigma)^{T} Q J_{w}(\sigma) \\
& \quad \leq \sigma \mu \int_{0}^{\sigma} w(s)^{T} E^{T}\left(\mathrm{e}^{A(\sigma-s)}\right)^{T}\left(\mathrm{e}^{A(\sigma-s)}\right) E w(s) d s \\
& \leq \sigma \mu \int_{0}^{\sigma} \mathrm{e}^{(\sigma-s) \lambda_{\max }\left(A+A^{T}\right)} w(s)^{T} E^{T} E w(s) d s \\
& \leq \sigma W \mu \lambda_{\max }\left(E^{T} E\right)\left(\int_{0}^{\sigma} \mathrm{e}^{\lambda_{\max }\left(A+A^{T}\right) s} d s\right)\|x\|^{2} \\
& \quad=\sigma W \mu \lambda_{\max }\left(E^{T} E\right) f_{A}(\sigma) x^{T} x,
\end{aligned}
$$

Implementing (38) and (39) in (37), it is clear that $G_{w}(\sigma, x) \leq x^{T} \Pi(\sigma) x$, with $\Pi(\sigma)$ defined in (7), and therefore, if $x^{T} \Pi(\sigma) x \leq 0$ for all $x \in \mathbb{R}^{n}$ and for all $\sigma \in\left[0, \tau_{\max }(x)\right]$, then the stability conditions from Lemma 9 are satisfied.

Proof of Lemma 3: Consider the constant sampling map $\tau_{\max }(x)=\tau^{*}, \forall x \in \mathbb{R}^{n}$. Since the sampling map is state-independent, we can remove the state-dependency in (6) by rewriting the inequality under the form of a parameter-dependent LMI: $\Pi(\sigma) \preceq 0, \forall \sigma \in\left[0, \tau^{*}\right]$. Then, the Schur complement ensures the equivalence between $\Pi(\sigma) \preceq 0$ and (11).

Proof of Theorem 4: Consider the constant sampling $\operatorname{map} \tau_{\max }(x)=\tau^{*}, \forall x \in \mathbb{R}$. (13) ensures that $\Delta(\sigma) \preceq 0$ for all $\sigma \in\left[0, \tau_{\max }(x)\right]$ if $\bar{\Delta}_{\kappa}\left(\tau^{*}\right) \preceq 0$ for all $\kappa \in \mathcal{K}\left(\tau^{*}\right)$. Using Lemma 3 , we can then show that the stability conditions from Theorem 2 are satisfied.

\section{Proof of Lemma 5:}

Step 1: Let us divide the time interval $[0, \bar{\sigma}]$ into $l$ subdivisions $\left[j \frac{\bar{\sigma}}{l},(j+1) \frac{\bar{\sigma}}{l}\right]$, with $j \in\{0, \cdots, l-1\}$. Let $\sigma \in\left[0, \sigma^{*}\right]$. There exists $j \in\left\{0, \cdots,\left\lfloor\frac{\sigma^{*} l}{\bar{\sigma}}\right\rfloor\right\}$ such that $j \frac{\bar{\sigma}}{l} \leq \sigma \leq(j+1) \frac{\bar{\sigma}}{l}$. Then define $\sigma^{\prime}=\sigma-j \frac{\bar{\sigma}}{l}\left(\sigma^{\prime} \in[0, \chi]\right.$, with $\chi=\frac{\bar{\sigma}}{l}$ if $j<\left\lfloor\frac{\sigma^{*} l}{\bar{\sigma}}\right\rfloor$, and $\chi=\sigma^{*}-\frac{j \bar{\sigma}}{l}$ otherwise). Step 2: In this step, we want to compute the Taylor expansion over $\left[j \frac{\bar{\sigma}}{l},(j+1) \frac{\bar{\sigma}}{l}\right]$ of the matrix function $\Delta$ defined in (11). It is possible to do so bloc by bloc. Therefore, we compute the Taylor expansion of $R, M_{2}^{T}$, and $M_{4}^{T}$, defined in (12) and (8). All three functions involve the term $\Lambda(\sigma)$ defined in (9). As in the unperturbed case
[8], we rewrite $\Lambda(\sigma)$ as a function of $\sigma^{\prime}$ :

$$
\begin{aligned}
\Lambda(\sigma) & =I+\left(N_{j}+\int_{0}^{\sigma^{\prime}} \mathrm{e}^{A s} d s N_{j}^{\prime}\right)(A-B K) \\
& =\Gamma_{1, j}+\sum_{i=1}^{\infty} \frac{A^{i-1}}{i !} \sigma^{i} \Gamma_{2, j},
\end{aligned}
$$

with the notations in (18). Therefore, one has

$$
\begin{aligned}
& M_{2}(\sigma)^{T}=-K^{T} B^{T} P+\Gamma_{1, j}^{T} M_{1}+\sum_{i=1}^{\infty} \Gamma_{2, j}^{T} \frac{\left(A^{i-1}\right)^{T}}{i !} M_{1} \sigma^{\prime i}, \\
& M_{4}(\sigma)^{T}=\Gamma_{1, j}^{T} P E+\sum_{i=1}^{\infty} \Gamma_{2, j}^{T} \frac{\left(A^{i-1}\right)^{T}}{i !} P E \sigma^{i} \\
& R(\sigma)=\sum_{k=0}^{\infty} L_{k, j} \sigma^{\prime k}
\end{aligned}
$$

with the matrices $L_{k, j}$ defined in (19). The matrices $\tilde{L}_{k, j}$ in (19) come from the Taylor expansion of the term $\sigma W \mu \lambda_{\max }\left(E^{T} E\right) f_{A}(\sigma) I$, and are defined in (20), (21). Using the Taylor expansion, one can show that $\Delta(\sigma)=$ $\sum_{k=0}^{\infty} \tilde{\Delta}_{(k, j)} \sigma^{\prime k}$, with the matrices $\tilde{\Delta}_{(k, j)}$ defined in $(17)$. A polynomial approximation of order $N$ of $\Delta$ on the interval $\left[j \frac{\bar{\sigma}}{l},(j+1) \frac{\bar{\sigma}}{l}\right]$ can therefore be expressed as

$$
\Xi_{(N, j)}\left(\sigma^{\prime}\right)=\sum_{k=0}^{N} \tilde{\Delta}_{(k, j)} \sigma^{\prime k}, \forall \sigma^{\prime} \in\left[0, \frac{\bar{\sigma}}{l}\right] .
$$

Step 3: The approximation error term $R_{(N, j)}\left(\sigma^{\prime}\right)=$ $\overline{\Delta(\sigma)}-\Xi_{(N, j)}\left(\sigma^{\prime}\right)$ can be bounded using the relation $R_{(N, j)}\left(\sigma^{\prime}\right) \preceq \nu I$, with $\nu$ a scalar defined in (22). With this, it is clear that if $\Xi_{(N, j)}\left(\sigma^{\prime}\right)+\nu I \preceq 0$, then $\Delta(\sigma) \preceq 0$. Step 4: Since the function $\Xi_{(N, j)}()+.\nu I:[0, \chi] \rightarrow$ $\overline{\mathcal{M}_{n}(\mathbb{R})}$ is polynomial, we can use the convex embedding design from [16], Section 3, to prove that if $\bar{\Delta}_{(i, j)}\left(\sigma^{*}\right) \preceq 0$ for all $i \in\{0, \cdots, N\}$, with $\bar{\Delta}_{(i, j)}\left(\sigma^{*}\right)=$ $\left(\sum_{k=0}^{i} \tilde{\Delta}_{(k, j)} \chi^{k}\right)+\nu I$, then $\Xi_{(N, j)}\left(\sigma^{\prime}\right)+\nu I \preceq 0$, and therefore $\Delta(\sigma) \preceq 0$.

Proof of Theorem 7: Let $x \in \mathbb{R}^{n}$. By the definition of the sampling map $\tau_{\text {max }}$ in $(26)$, we have $x \in \hat{\mathcal{R}}\left(\tau_{\max }(x)\right)$. Since it is assumed that $\hat{\mathcal{R}}(\tilde{\sigma}) \subset \mathcal{R}(\tilde{\sigma})$ for all $\tilde{\sigma} \in[0, \bar{\sigma}]$, and $\tau_{\max }(x) \in \Upsilon \subset[0, \bar{\sigma}]$, we have $x \in \mathcal{R}\left(\tau_{\max }(x)\right)$, and thus the condition (6) from Theorem 2 is satisfied. The other assumptions and conditions guarantee that all the stability conditions from Theorem 2 are satisfied.

Proof of Theorem 8: Consider scalars $\rho_{s} \geq 0$ such that the LMI conditions (33a) are satisfied for all $s \in$ $\{1, \cdots, q\}$. Let $x \in \mathbb{R}^{n}$. There exists $s \in\{1, \cdots, q\}$ such that $x \in \mathcal{R}_{s}$. According to (33), one has $\Delta(\sigma)+$ $\left[\begin{array}{cc}\rho_{s} \Phi_{s} & 0 \\ * & 0\end{array}\right] \preceq 0$ for all $\sigma \in\left[0, \tau_{\max }^{(s)}\right]$. Thus, using the construction of $\Delta$ (equation (11)) and the Schur complement, we get that $\Pi(\sigma)+\rho_{s} \Phi_{s} \preceq 0$ for all $\sigma \in\left[0, \tau_{\max }^{(s)}\right]$, 
with $\Pi$ defined in (7). Since $x \in \mathcal{R}_{s}=\left\{x \in \mathbb{R}^{n}, x^{T} \Phi_{s} x \geq\right.$ $0\}$, the S-procedure then ensures that $x^{T} \Pi(\sigma) x \preceq 0$ for all $\sigma \in\left[0, \tau_{\max }^{(s)}=\tau_{\max }(x)\right]$, and thus, the conditions from Theorem 2 are satisfied.

\section{References}

[1] A. Anta and P. Tabuada. To sample or not to sample: selftriggered control for nonlinear systems. IEEE Transactions on Automatic Control, 55(9):2030-2042, 2010.

[2] A. Anta and P. Tabuada. Exploiting isochrony in selftriggered control. IEEE Transactions on Automatic Control, 57(4):950-962, 2012.

[3] K.-E. Arzn. A simple event-based pid controller. In 14th IFAC World Congress, pages 423-428, Beijing, China, 1999.

[4] C. Briat and A. Seuret. Robust stability of impulsive systems: a functional-based approach. In 4th IFAC conference on Analysis and Design of Hybrid Systems, Eindhoven, Netherlands, 2012.

[5] M.B.G. Cloosterman, L. Hetel, N. van de Wouw, W.P.M.H. Heemels, J. Daafouz, and H. Nijmeijer. Controller synthesis for networked control systems. Automatica, 46(10):15841594, 2010.

[6] M.C.F. Donkers and W.P.M.H. Heemels. Outputbased event-triggered control with guaranteed $\mathcal{L}_{\infty}$-gain and improved and decentralized event-triggering. IEEE Transactions Automatic Control, 57(6):1362-1376, 2012.

[7] C. Fiter, L. Hetel, W. Perruquetti, and J.-P. Richard. State dependent sampling: an LMI based mapping approach. In 18th IFAC World Congress, pages 8824-8829, Milan, Italy, 2011.

[8] C. Fiter, L. Hetel, W. Perruquetti, and J.-P. Richard. A state dependent sampling for linear state feedback. Automatica, 48(8):1860-1867, 2012.

[9] C. Fiter, L. Hetel, W. Perruquetti, and J.-P. Richard. Statedependent sampling for perturbed time-delay systems. In 51st IEEE Conference on Decision and Control, pages 23582363, Maui, Hawaii, USA, 2012.

[10] C. Fiter, H. Omran, L. Hetel, and J.-P. Richard. Tutorial on arbitrary and state-dependent sampling. In 13th European Control Conference, pages 1440-1445, Strasbourg, France, 2014.

[11] E. Fridman. A refined input delay approach to sampled-data control. Automatica, 46(2):421-427, 2010.

[12] E. Fridman, A. Seuret, and J.-P Richard. Robust sampleddata stabilization of linear systems: An input delay approach. Automatica, 40(8):1441-1446, 2004.

[13] H. Fujioka. Stability analysis of systems with aperiodic sample-and-hold devices. Automatica, 45(3):771-775, 2009.

[14] K. Gu, V. Kharitonov, and J. Chen. Stability of time-delay systems. Boston: Birkhauser, 2003.

[15] W.P.M.H. Heemels, M.C.F. Donkers, and A.R. Teel. Periodic event-triggered control for linear systems. IEEE Transactions Automatic Control, 58(4):847-861, 2013.

[16] L. Hetel, J. Daafouz, and C. Iung. Stabilization of arbitrary switched linear systems with unknown time-varying delays. IEEE Transactions on Automatic Control, 51(10):1668-1674, 2006.

[17] L. Hetel, A. Kruszewski, W. Perruquetti, and J.-P Richard. Discrete and intersample analysis of systems with aperiodic sampling. IEEE Transactions on Automatic Control, 56(7):1696-1701, 2011.
[18] I. Karafyllis and C. Kravaris. Global stability results for systems under sampled-data control. International Journal of Robust and Nonlinear Control, 19(10):1105-1128, 2009.

[19] V. Kolmanovskii and A. Myshkis. Applied theory of functional differential equations. Springer, 1992.

[20] K. Liu and E. Fridman. Wirtinger's inequality and Lyapunovbased sampled-data stabilization. Automatica, 48(1):102108, 2012.

[21] K. Liu, V. Suplin, and E. Fridman. Stability of linear systems with general sawtooth delay. IMA Journal of Mathematical Control and Information, 27(4):419-436, 2010.

[22] C.-V Loan. The sensitivity of the matrix exponential. SIAM Journal on Numerical Analysis, 14(6):971-981, 1977.

[23] J. Lunze and D. Lehmann. A state-feedback approach to event-based control. Automatica, 46(1):211-215, 2010.

[24] J.-L. Mancilla-Aguilar, R. Garcia, E. Sontag, and Y. Wang. On the representation of switched systems with inputs by perturbed control systems. Nonlinear Analysis: Theory, Methods and Applications, 60(6):1111-1150, 2005.

[25] M. Mazo Jr., A. Anta, and P. Tabuada. An ISS selftriggered implementation of linear controllers. Automatica, 46(8):1310-1314, 2010.

[26] L. Mirkin. Some remarks on the use of time-varying delay to model sample-and-hold circuits. IEEE Transactions on Automatic Control, 52(6):1109-1112, 2007.

[27] D. Nesic, A. Teel, and D. Carnevale. Explicit computation of the sampling period in emulation of controllers for nonlinear sampled-data systems. IEEE Transactions on Automatic Control, 54(3):619-624, 2009.

[28] H. Omran, L. Hetel, J.-P. Richard, and F. LamnabhiLagarrigue. Stability analysis of bilinear systems under aperiodic sampled-data control. Automatica, 50(4):12881295, 2014.

[29] C. Peng and T.-C. Yang. Event-triggered communication and $h_{\infty}$ control co-design for networked control systems. Automatica, 49(5):1326-1332, 2013.

[30] R. Postoyan, A. Anta, W.P.M.H. Heemels, P. Tabuada, and D. Nesic. Periodic event-triggered control for nonlinear systems. In 52th IEEE Conference on Decision and Control, Florence, Italy, 2013.

[31] R. Postoyan, A. Anta, D. Nesic, and P. Tabuada. A unifying Lyapunov-based framework for the event-triggered control of nonlinear systems. In 50th IEEE Conference on Decision and Control, pages 2559-2564, Orlando, Florida, USA, 2011.

[32] A. Seuret. A novel stability analysis of linear systems under asynchronous samplings. Automatica, 48(1):177-182, 2012.

[33] P. Tabuada. Event-triggered real-time scheduling of stabilizing control tasks. IEEE Transactions on Automatic Control, 52(9):1680-1685, 2007.

[34] A. Teel, D. Nesic, and P. Kokotovic. A note on input-tostate stability of nonlinear sampled-data systems. In $37 t h$ IEEE Conference on Decision and Control, pages 2473-2478, Tampa, Florida, USA, 1998.

[35] X. Wang and M.-D. Lemmon. Self-triggered feedback control systems with finite-gain $\mathcal{L}_{2}$ stability. IEEE Transactions on Automatic Control, 54(3):452-467, 2009.

[36] X. Wang and M.-D. Lemmon. Self-triggering under stateindependent disturbances. IEEE Transactions on Automatic Control, 55(6):1494-1500, 2010.

[37] D. Yue, E. Tian, and G.-L. Han. A delay system method for designing event-triggered controllers of networked control systems. IEEE Transactions on Automatic Control, 58(2):475-481, 2013. 